\title{
Role of serum biomarkers to optimise a validated clinical risk prediction tool for
}

Gestational Diabetes

\section{Short title: Optimising GDM clinical risk prediction tools}

Sally K ABELL ${ }^{1}$, Soulmaz SHORAKAE ${ }^{2}$, Jacqueline A BOYLE ${ }^{3}$, Barbora DE COURTEN ${ }^{4}$, Nigel K STEPTO 5 , Helena J TEEDE ${ }^{6}$ and Cheryce L HARRISON ${ }^{7}$.

${ }^{1}$ Post-doctoral researcher. Monash Centre for Health Research and Implementation, School of Public Health and Preventive Medicine, Monash University, Clayton, Victoria, Australia; Endocrinologist, Diabetes and Vascular Medicine Unit, Monash Health, Clayton, Victoria, Australia. Email: sally.abell@monash.edu

2 Doctoral Candidate. Monash Centre for Health Research and Implementation, School of Public Health and Preventive Medicine, Monash University, Clayton, Victoria, Australia; Endocrinologist, Diabetes and Vascular Medicine Unit, Monash Health, Clayton, Victoria, Australia. Email: soulmaz.shorakae@monash.edu

${ }^{3}$ Head of Refugee and Indigenous Women's Health, Monash Centre for Health Research and Implementation, School of Public Health and Preventive Medicine, Monash University, Clayton, Victoria, Australia; Obstetrician \& Gynaecologist, Monash Women's Services, Monash Health, Clayton, Victoria, Australia. Email: jaqueline.boyle@ monash.edu

${ }^{4}$ Head of Chronic Disease Prevention, Monash Centre for Health Research and Implementation, School of Public Health and Preventive Medicine, Monash University, Clayton, Victoria, Australia; General Physician, Diabetes and Vascular Medicine Unit, Monash Health, Clayton, Victoria, Australia. Email: barbora.decourten@monash.edu ${ }^{5}$ Professor of Clinical Exercise Science, Institute of Sport, Exercise and Active Living, Victoria University, Footscray, Victoria. Email: nigel.stepto@vu.edu.au

${ }^{6}$ Director Monash Centre for Health Research and Implementation, School of Public Health and Preventive Medicine, Monash University, Clayton, Victoria, Australia; Professor of Diabetes, Diabetes and Vascular Medicine Unit, Monash Health, Clayton, Victoria, Australia. Email: helena.teede@monash.edu

${ }^{7}$ Research Fellow, Monash Centre for Health Research and Implementation, School of Public Health and Preventive Medicine, Monash University, Clayton, Victoria, Australia. Email: cheryce.harrison@monash.edu

This is the author manuscript accepted for publication and has undergone full peer review but has not been through the copyediting, typesetting, pagination and proofreading process, which may lead to differences between this version and the Version of Record. Please cite this article as doi: $10.1111 / a j o .12833$

This article is protected by copyright. All rights reserved 


\section{*Corresponding Author}

Dr Cheryce Harrison, PhD AEP

National Heart Foundation Research Fellow

Monash Centre for Health Research and Implementation

School of Public Health and Preventive Medicine

Locked Bag 29 Clayton, VIC 3168, Australia.

ph: 85722662

\section{The authors have no conflicts of interest}

\section{Acknowledgements}

SKA, SS, JAB, BdC, NKS, HJT and CLH planned and designed the study. SKA and SS performed laboratory biomarker analysis. SKA and CLH drafted the manuscript and SKA performed the data analysis. All authors provided significant intellectual contribution in interpreting data and critical review of the manuscript. All authors approved the final version of the manuscript. The authors would like to acknowledge Ms Danielle Hiam, Dr Alba Moreno-Asso, Dr Samantha Cassar and Ms Tracy Murray from Victoria University for assisting with adipocytokine analysis.

\section{Funding}

This work was supported by an ISEAL Victoria University Clinical Exercise Science seed funding grant. The funder was not involved in study design, analysis or interpretation.

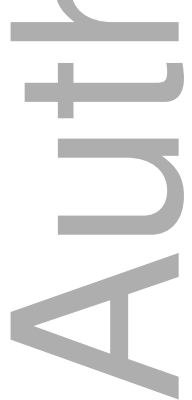


DR. SALLY K ABELL (Orcid ID : 0000-0002-3154-4946)

PROF. HELENA J TEEDE (Orcid ID : 0000-0001-7609-577X)

Article type : Original Manuscript

西

Role of serum biomarkers to optimise a validated clinical risk prediction tool for Gestational Diabetes

Short title: Optimising GDM clinical risk prediction tools

Word Counts

Abstract 250

Main text 2618

Tables 2

Figures 2

Keywords:

biomarkers, adipocytokines, risk prediction, gestational diabetes

\section{Conflicts of Interest Statement}

There are no conflicts of interest.

Role of serum biomarkers to optimise a validated clinical risk prediction tool for Gestational Diabetes

\section{Background}

Clinical risk prediction tools for gestational diabetes (GDM) may be enhanced by measuring biomarkers in early pregnancy.

\section{Aim}

Evaluate a two-step GDM risk prediction tool incorporating fasting glucose (FG) and serum

This article is protected by copyright. All rights reserved 
biomarkers in early pregnancy.

\section{Materials and Methods}

High molecular weight (HMW) adiponectin, omentin-1 and interleukin-6 (IL-6) were measured at $12-15$ weeks gestation in women from a randomized trial with high risk of GDM using a clinical risk prediction tool. GDM diagnosis (24-28 weeks) was evaluated using 1998 Australian Diabetes in Pregnancy (ADIPS) criteria and newer International Association of the Diabetes and Pregnancy Study Groups (IADPSG) criteria.

Associations between biomarkers and development of GDM were examined using multivariable regression analysis. Area under the receiver-operator curve (AUC), sensitivity and specificity were calculated to determine classification ability of each model compared to FG and maternal characteristics.

\section{Results}

HMW adiponectin improved prediction of ADIPS GDM (AUC 0.85, sensitivity 50\%, specificity $96.2 \%, \mathrm{p}=0.04)$, compared to $\mathrm{FG}$ and maternal factors $(0.78,35 \%, 98.1 \%$ respectively). HMW adiponectin $<1.53 \mu \mathrm{g} / \mathrm{mL}$ further improved the model (AUC 0.87, sensitivity $75 \%$, specificity $88.2 \%, \mathrm{p}=0.01$ ).

HMW adiponectin did not improve prediction of IADPSG GDM (AUC 0.84, sensitivity 64\%, specificity $97.9 \%, \mathrm{p}=0.22)$ compared to $\mathrm{FG}$ and maternal factors $(0.79,56 \%, 93.8 \%)$.

Omentin-1 and IL-6 did not significantly improve classification ability for GDM.

\section{Conclusions}

A two-step approach combining FG and HMW adiponectin to a validated clinical risk prediction tool improved sensitivity and predictive ability for ADIPS GDM. Further research is required to enhance GDM prediction using IADPSG criteria for application in clinical practice. 


\section{Role of serum biomarkers to optimise a validated clinical risk prediction tool for Gestational Diabetes}

\section{Introduction}

The prevalence of Gestational Diabetes Mellitus (GDM) is increasing due to advanced maternal age, increasing proportions of women entering pregnancy overweight or obese ${ }^{1}$ and implementation of the International Association of the Diabetes and Pregnancy Study Groups (IADPSG) diagnostic criteria. ${ }^{2}$ Gestational Diabetes Mellitus (GDM) is associated with adverse maternal and neonatal outcomes, ${ }^{3}$ and treatment of GDM reduces these risks. ${ }^{4,5}$ Following pregnancy, women with a history of GDM and their offspring have higher long term risk of obesity, type 2 diabetes mellitus (T2DM) and cardiovascular disease. ${ }^{6,7}$

With a primary focus on GDM diagnosis and treatment, opportunities for prevention of GDM are often missed. ${ }^{1}$ Universal testing for GDM is recommended at $24-28$ weeks. ${ }^{2,8,9}$ Yet simple, early screening algorithms based on maternal risk factors may identify and reliably predict women at high risk of GDM, before glucose intolerance is detectable. ${ }^{10}$ Early identification of women at high risk of GDM enables streamlined antenatal care, may enhance continuity of care, facilitates implementation of potential GDM preventive measures, prompts timely screening and diagnosis, and optimises management to reduce adverse outcomes if GDM occurs. ${ }^{11,12}$ Furthermore, early identification of women at risk can inform the basis of randomized controlled trials (RCTs) to guide targeted future prevention strategies for GDM. ${ }^{13}$

Our group developed and validated a clinical first trimester risk prediction tool to identify women at risk of GDM. ${ }^{1}$ A simple scoring tool based on clinical risk factors achieved $83.9 \%$ and $61.3 \%$ sensitivity and $39.0 \%$ and $71.4 \%$ specificity for detection of GDM at scores of 3 and 4 respectively (Table 1 ). ${ }^{1}$ The tool was then validated internationally showing sensitivity of $65.6 \%$, specificity $75.0 \%$ and area under curve (AUC) of 0.74 for GDM diagnosis. ${ }^{10} \mathrm{We}$ then applied this tool to screen 1331 women for recruitment into an early pregnancy healthy 
lifestyle RCT (HeLP-her HRP). ${ }^{14,15}$ We demonstrated a significant reduction in excess gestational weight gain and postpartum weight retention, yet were not powered to reduce incidence of GDM. ${ }^{14,15}$ However, meta-analyses of combined studies with increased participant numbers do suggest GDM can be prevented with early lifestyle intervention. ${ }^{16}$

Subsequently, in the context of the HeLP-her HRP study, we examined addition of fasting biochemical markers (glucose and lipids) to the GDM risk prediction tool. ${ }^{11}$ Women were diagnosed with GDM based on Australasian Diabetes in Pregnancy Society (ADIPS) 1998 criteria, ${ }^{17}$ which were in use at the time, and also on proposed IADPSG criteria. ${ }^{2}$ Incorporating fasting glucose at 12-15 weeks gestation incrementally improved the clinical risk prediction tool with sensitivity and specificity of $34 \%$ and $94.8 \%$ for ADIPS-diagnosed GDM and 50\% and $92.4 \%$ for IADPSG-diagnosed GDM. ${ }^{11}$

Recently, we examined novel serum adipocytokines and inflammatory markers at 12-15 weeks in this cohort. ${ }^{18}$ When combined with maternal factors including age, body mass index (BMI) and past history of GDM, development of GDM was associated with levels of high molecular weight (HMW) adiponectin, omentin-1 and interleukin-6 (IL-6). In the current analysis, we aim to optimise the validated risk prediction tool with fasting glucose and add these novel biomarkers in attempt to improve sensitivity and predictive ability for GDM. If these biomarkers can further enhance discriminative ability of the tool, their use may improve early prediction of GDM in a two step approach in women clinically identified at risk, thus allowing intervention to reduce risk of GDM and related adverse pregnancy outcomes.

\section{Materials and Methods}

Study design and population

The study population was taken from the HeLP-her HRP RCT, aiming for the prevention of excess gestational weight gain (GWG) in women at risk of GDM. ${ }^{13}$ Study participants were identified as being high GDM risk (score $\geq 3$ ) based on our validated risk prediction tool. ${ }^{8}$ The study was approved by the Monash Health Research Advisory and Ethics Committee (Project no. 07216C 1/4/2008) and methods have been published previously. ${ }^{14}$ Recruitment occurred at 3 tertiary hospitals within Monash Health in Melbourne, Australia (2009-2010) and captured 228 women with singleton pregnancies < 15 weeks gestation if overweight $\left(\mathrm{BMI}>25 \mathrm{~kg} / \mathrm{m}^{2}\right.$ or $>23 \mathrm{~kg} / \mathrm{m}^{2}$ if high risk ethnicity) or obese $\left(\mathrm{BMI} \geq 30 \mathrm{~kg} / \mathrm{m}^{2}\right)$. Exclusion 
criteria included $\mathrm{BMI} \geq 45 \mathrm{~kg} / \mathrm{m}^{2}$, non-English speaking, known pre-existing diabetes or a chronic medical condition.

This current study examines the pregnant women allocated to the control arm of the HeLPher HRP RCT with biobanked serum available for analysis $(n=103)$. These women received one non-interactive session providing information and advice on appropriate health lifestyles (diet and physical activity). Demographic characteristics were collected on a baseline questionnaire, and included maternal age, country of birth, past history of GDM and family history of diabetes in a first degree relative. Body mass index (BMI) was calculated at the baseline visit (12-15 weeks).

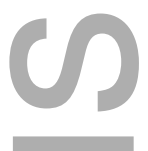

Biochemical and biomarker measurements

Biobanked serum samples consisted of fasting venous blood drawn at 12-15 weeks gestation. Serum was stored in multiple aliquots under sterile conditions at $-80^{\circ} \mathrm{C}$. Fasting glucose (FG) was measured on a Beckman Coulter LX20PRO Analyser using a commercial kit (Beckman Coulter Diagnostics, Australia) as described previously. ${ }^{11}$

Sandwich-type enzyme linked immunosorbent assays (ELISA) were used to measure biomarkers. HMW adiponectin was measured using the ALPCO ELISA (Cat no. 47ADPHU-E01) with a sensitivity of $0.019 \mathrm{ng} / \mathrm{mL}$. IL-6 was measured using the Abcam high sensitivity ELISA (ab46042) with sensitivity 0.81pg/mL. Omentin-1 was measured using the Millipore ELISA (EZHOMNTN1-29K) with a sensitivity of 0.23ng/mL. Samples were thawed prior to use and all assays were run in duplicate according to the manufacturers' instructions. Intrassay and interassay coefficients of variation were all $<10 \%$ and methods have been described previously. ${ }^{18}$ Analyte concentrations were derived from best-fit standard curves.

GDM testing

Development of GDM was the study outcome. Ninety (87\%) of the control women completed GDM testing at 26-28 weeks gestation with an oral glucose tolerance test. Diagnosis of GDM was based on both ADIPS 1998 criteria $^{17}$ (fasting plasma glucose 
$\geq 5.5 \mathrm{mmol} / \mathrm{L}$ and $/$ or $2 \mathrm{hr} \geq 8.0 \mathrm{mmol} / \mathrm{L}$ ) and $\mathrm{IADPSG}$ criteria $^{2}$ (fasting $\geq 5.1 \mathrm{mmol} / \mathrm{L}$, and $/$ or $1 \mathrm{hr}$ $\geq 10 \mathrm{mmol} / \mathrm{L}$ and/or $2 \mathrm{hr} \geq 8.5 \mathrm{mmol} / \mathrm{L}$ ). Thirteen women (13\%) mistakenly underwent a screening $50 \mathrm{~g}$ glucose challenge test (GCT) as per standard practice at the time, and did not progress to OGTT per the study protocol.

\section{Statistical Analysis}

Statistical methods have been described previously. ${ }^{11}$ The association between each biomarker and development of GDM was evaluated using univariable and multivariable logistic regression analysis and reported as crude and adjusted odds ratios (OR) with $95 \%$ confidence intervals (CI). Maternal factors from the risk prediction tool were included as covariates in multivariable models: maternal age, BMI, ethnicity, past history of GDM, and family history of diabetes. Firstly, fasting glucose was evaluated as a covariate in addition to maternal factors in our study population (as published previously ${ }^{11}$ ). Area under the curve (AUC), sensitivity and specificity were calculated to determine the classification ability of each model, using the fasting glucose and maternal factors model as the comparator. Each biomarker was separately added to these models to determine the incremental prediction ability for GDM. To evaluate the predictive accuracy of each marker for GDM, we generated receiver operating characteristic (ROC) curves from multivariable logistic regression models. ROC comparison testing was used to determine statistical significance of each model against the original model. The same method was then used to analyse biomarker results in quartiles. The analysis was performed using STATA 14 (StataCorp, Texas, USA) and two-sided pvalues were significant at $\mathrm{p}<0.05$.

\section{Results}

Biomarkers that may be predictive of GDM

In univariable regression analysis, FG was associated with increased risk of ADIPS GDM with an OR of 5.47 (95\% CI 1.75-17.10) and IADPSG GDM with an OR of 12.64 (3.3847.23). HMW adiponectin was associated with a reduced risk of ADIPS GDM 0.40 (0.220.71 ) and IADPSG GDM 0.53 (0.34-0.82). Interleukin-6 and omentin-1 were not associated with GDM on univariable analysis, but were incorporated into this current analysis based on our previous data showing associations with ADIPS GDM when adjusted for maternal factors. $^{17}$

Risk prediction tool incorporating new biomarkers 
Results for FG and biomarkers in combination with maternal factors from the risk prediction tool are reported in Table 2. FG and maternal factors resulted in an AUC of 0.78 with sensitivity $35 \%$ and specificity of $98.1 \%$ for prediction of ADIPS GDM and AUC 0.79 with sensitivity $56 \%$ and specificity $93.8 \%$ for prediction of IADPSG GDM. For prediction of ADIPS GDM, the addition of HMW adiponectin improved AUC to 0.85 with increased sensitivity of $50 \%$ and a fall in specificity to $96.2 \%$ (Figure 1a). The addition of omentin-1 improved AUC to 0.84 with increase in sensitivity to $44.4 \%$ and specificity reduced to $96.2 \%$. The addition of IL-6 resulted in a decrease in AUC to 0.77, with improved sensitivity to $50.0 \%$ and reduced specificity to $78.0 \%$. The improvement in classification ability over the FG model was statistically significant for adiponectin ( $\mathrm{p}=0.04)$. When HMW adiponectin was further evaluated in quartiles, the lowest quartile of HMW adiponectin $<1.53 \mu \mathrm{g} / \mathrm{mL}$ resulted in further improved AUC of 0.87 , with sensitivity of $75 \%$ and specificity of $88.2 \%$ ( $\mathrm{p}=0.01$ ).

For prediction of IADPSG GDM, addition of HMW adiponectin improved AUC to 0.84 with increased sensitivity of $64 \%$ and specificity of $97.9 \%$, but was not statistically significant compared to the FG model ( $\mathrm{p}=0.22$ ) (Figure $1 \mathrm{~b}$ ). HMW adiponectin $<1.53 \mu \mathrm{g} / \mathrm{mL}$ did not significantly improve the FG model (AUC 0.85, sensitivity 64\%, specificity 95.7\%, p=0.43). The other markers did not significantly improve classification ability for IADPSG GDM.

\section{Discussion}

Accurate early identification of women at high risk of GDM provides opportunity to streamline antenatal care models and apply future interventions to reduce development of GDM and associated healthcare costs and adverse outcomes. ${ }^{11,19}$ Clinical screening tools have moderate predictive value and appear improved by the addition of fasting glucose, however incremental value with biomarkers of insulin resistance and inflammation that precede glucose intolerance remain unclear. ${ }^{20,21}$ We build on our past publication noting improved GDM prediction after combining fasting glucose at 12-15 weeks gestation with our validated clinical risk prediction tool in women identified at high risk of GDM. ${ }^{11}$ Here, we expand this risk prediction tool with addition of adipocytokines HMW adiponectin, omentin1 and IL-6 (measured at 12-15 weeks of pregnancy), which we have shown are associated with GDM. ${ }^{18}$ The addition of HMW adiponectin to fasting glucose and maternal factors resulted in significantly improved sensitivity and classification ability for ADIPS GDM, in particular with HMW adiponectin less than $1.53 \mu \mathrm{g} / \mathrm{mL}$. Incorporating omentin-1 resulted in a 
modest improvement in prediction of ADIPS GDM, however this was not statistically significant. The addition of IL-6 did not incrementally improve model prediction. The addition of adipocytokines to the IADPSG GDM models did not improve model prediction.

Low first trimester adiponectin may predict development of $\mathrm{GDM}^{22,23}$ and the performance of clinical screening tools may be incrementally improved by adiponectin levels. ${ }^{25,26}$ Rasanen et al. (2013) found that first trimester adiponectin (5-13 weeks) had modest classification performance (AUC 0.63) for detection of GDM (diagnosed if fasting, $1 \mathrm{hr}$ or $2 \mathrm{hr}$ glucose values exceeded $5.3 \mathrm{nmol} / \mathrm{L}, 10.0 \mathrm{nmol} / \mathrm{L}$ and $8.6 \mathrm{nmol} / \mathrm{L}$ respectively) after adjustment for age, gestational age at sample collection and nulliparity. ${ }^{26}$ Notably, they did not report BMI data in their cohort, which may over or underestimate risk. Nanda et al. reported a detection rate of $61.6 \%$ (AUC 0.79 , false positive rate $20 \%$ ) in screening for GDM by maternal characteristics (age, BMI, race, previous history of GDM and macrosomic neonate), which improved to $68.7 \%$ (AUC 0.83 ) by addition of adiponectin at $11-13$ weeks. ${ }^{25}$ However, GDM testing was performed with outdated World Health Organization 1999 diagnostic criteria. Here, we examined HMW adiponectin, the isoform most responsible for metabolic effects. $^{27,28}$ We propose the further addition of HMW adiponectin in a two-step approach in women identified at high risk based on maternal clinical risk factors, thereby increasing sensitivity from $35 \%$ to $50 \%$ and improving classification ability from $78 \%$ to $85 \%$ for ADIPS GDM compared to fasting glucose and maternal clinical factors alone. Sensitivity was substantially improved to $75 \%$ when HMW adiponectin level was less than $1.53 \mu \mathrm{g} / \mathrm{mL}$, at the expense of a higher false positive rate (12\% versus $2 \%)$. HMW adiponectin also improved the risk prediction tool using IADPSG criteria for GDM, with a higher AUC of 0.84 , improved sensitivity $64 \%$ and specificity $97.9 \%$ compared to fasting glucose and maternal factors (AUC 0.79, sensitivity 56\%, specificity 93.8\%). However, this was not statistically significant. HMW adiponectin appears to be a good predictor of later GDM development that improves classification ability for ADIPS GDM in our risk prediction tool, beyond that reported in other published tools. However, HMW adiponectin does not improve prediction of the more recently adopted IADPSG criteria. Further prospective studies are required to validate these findings and determine cost-effectiveness in the clinical setting.

Interleukin-6 is reported to be increased in women with GDM, and is correlated with insulin sensitivity and BMI. ${ }^{29}$ Hassiakos et al. (2015) found that first trimester (11-14weeks) levels 
of IL-6 may predict IADPSG GDM (AUC 0.67) in a low risk population, and when combined with maternal weight and age (AUC 0.76). ${ }^{30}$ Here, in addition to fasting glucose and other maternal factors in a high-risk population, IL-6 improved sensitivity of our risk prediction tool, but reduced AUC for ADIPS GDM (0.78 to 0.77) and significantly reduced specificity (98.1\% to 78\%). IL-6 did not improve prediction of IADPSG GDM. IL-6 may be a less useful early marker for GDM prediction, as the relatively small changes in insulin resistance in early pregnancy may be insufficient to significantly alter levels. ${ }^{22}$

Omentin-1 has been linked to susceptibility to type 2 diabetes, and may be dysregulated in GDM. We previously reported that omentin-1 was significantly associated with ADIPS GDM on multivariable analysis, after adjustment for maternal factors including BMI. ${ }^{18}$ Omentin-1 improved our risk prediction tool with AUC 0.84 , sensitivity of $44.4 \%$ but reduced specificity to $96.2 \%$, however this was not statistically significant compared to the fasting glucose and maternal factors model. Thus, it appears that omentin-1 is not as strong a predictor of GDM as HMW adiponectin, and it does not significantly improve classification ability.

This study builds on our validated clinical risk prediction tool in pregnancy, with which we demonstrated good sensitivity and specificity for identifying women who developed GDM in the context of a large scale antenatal lifestyle intervention trial. Here, we show that addition of fasting glucose and HMW adiponectin, in combination with maternal factors, can substantially improve predictive value of our model for ADIPS GDM, with high accuracy, moderate sensitivity and high specificity. Notably, results using our tool for IADPSG GDM were less impressive than ADIPS GDM, thus significantly compromising its clinical application. We speculate that this reflects the milder spectrum of GDM identified by IADPSG criteria, noting higher median HMW adiponectin levels seen in women with IADPSG GDM in our original paper. ${ }^{18}$ During the study period, GDM screening in early pregnancy (12-15 weeks gestation) was not routine. As serum was collected at baseline (1215 weeks) for research purposes and examined retrospectively, women with elevated fasting glucose in the range that would now be considered early GDM may have been included in the analysis. Other limitations of our study include that the ELISAs used were research quality valid, and larger scale studies are required to standardize this assay, reduce cost and improve accessibility. Furthermore, sensitivities and specificities have been derived from a group of women at high risk of GDM based on clinical factors, thus utility of biomarkers in risk prediction tools including all pregnant women remains unknown. 
The ideal multiparametric risk prediction tool for GDM would incorporate clinical risk factors and biomarkers that precede the onset of hyperglycaemia, thus potentially avoiding harm caused by overt glucose intolerance in late pregnancy. ${ }^{31}$ To translate to clinical practice, it must be easily administered by the clinician, acceptable to the patient, and it must also be cost-effective with resources available to support its use, and demonstration of improved outcomes in those deemed high risk. The challenge is to find a tool which improves accuracy, whilst being affordable, practical and clinically feasible. ${ }^{11}$ Fasting glucose is commonly measured in early pregnancy to screen high risk women and is likely to be cost effective. At this stage, in considering cost benefit, incremental value over the simple fasting glucose model, and lack of improvement in models examining IADPSG criteria, our results show that biomarkers do not improve models sufficiently to warrant clinical application. However, HMW adiponectin may be a promising marker to improve classification ability and sensitivity of prediction tools in a two-step approach. Prospective studies are required using larger sample sizes and IADPSG criteria. Thereafter, cost effectiveness, reduction in GDM and improved clinical outcomes beyond simple clinical models must be demonstrated.

Use of a two-step approach with a validated clinical risk prediction tool and in those at risk, addition of HMW adiponectin and fasting glucose improved sensitivity and predictive ability for ADIPS GDM. However, further research is required to prospectively study and validate this more complex tool for clinical practice, and using IADPSG diagnostic criteria.

\section{References}

1. Teede HJ, Harrison CL, Teh WT, Paul E, Allan CA. Gestational diabetes: development of an early risk prediction tool to facilitate opportunities for prevention. Aust $\mathrm{N}$ Z J Obstet Gynaecol. 2011;51(6):499-504.

2. Metzger BE, Gabbe SG, Persson B, Buchanan TA, Catalano PA, Damm P, et al. International association of diabetes and pregnancy study groups recommendations on the diagnosis and classification of hyperglycemia in pregnancy. Diabetes Care. 2010;33(3):676682.

3. Metzger BE, Lowe LP, Dyer AR, Trimble ER, Chaovarindr U, et al. Hyperglycemia and adverse pregnancy outcomes. N Engl J Med. 2008;358(19):1991-2002.

4. Landon MB, Spong CY, Thom E, Carpenter MW, Ramin SM, Casey B, et al. A multicenter, randomized trial of treatment for mild gestational diabetes. N Engl J Med. 2009;361(14):1339-1348. 
5. Crowther CA, Hiller JE, Moss JR, McPhee AJ, Jeffries WS, Robinson JS. Effect of treatment of gestational diabetes mellitus on pregnancy outcomes. N Engl J Med. 2005;352(24):2477-2486.

6. Silverman BL, Rizzo T, Green OC, Cho NH, Winter RJ, Ogata ES, et al. Long-term prospective evaluation of offspring of diabetic mothers. Diabetes. 1991;40 Suppl 2:121-125. 7. Vrachnis N, Augoulea A, Iliodromiti Z, Lambrinoudaki I, Sifakis S, Creatsas G. Previous gestational diabetes mellitus and markers of cardiovascular risk. Int J Endocrinol. 2012;2012:458610. doi: 10.1155/2012/458610.

8. Nankervis A, McIntyre HD, Moses R, Ross GP, Callaway L, Porter C, et al. ADIPS consensus guidelines for the testing and diagnosis of gestational diabetes mellitus in Australia. 2014. Available at: https://www.ranzcog.edu.au/doc/adips-gdm-guidelines.html. 9. Teh WT, Teede HJ, Paul E, Harrison CL, Wallace EM, Allan C. Risk factors for gestational diabetes mellitus: implications for the application of screening guidelines. Aust $\mathrm{N}$ Z J Obstet Gynaecol. 2011;51(1):26-30.

10. Theriault S, Forest JC, Masse J, Giguere Y. Validation of early risk-prediction models for gestational diabetes based on clinical characteristics. Diabetes Res Clin Pract. 2014;103(3):419-425.

11. Harrison CL, Lombard CB, East C, Boyle J, Teede HJ. Risk stratification in early pregnancy for women at increased risk of gestational diabetes. Diabetes Res Clin Pract. 2015;107(1):61-68.

12. Nicolaides KH. A model for a new pyramid of prenatal care based on the 11 to 13 weeks' assessment. Prenat Diagn. 2011;31(1):3-6.

13. Eleftheriades M, Papastefanou I, Lambrinoudaki I, Kappou D, Lavranos D, Akalestos A, et al. Elevated placental growth factor concentrations at 11-14 weeks of gestation to predict gestational diabetes mellitus. Metabolism. 2014;63(11):1419-1425.

14. Harrison CL, Lombard CB, Strauss BJ, Teede HJ. Optimizing healthy gestational weight gain in women at high risk of gestational diabetes: a randomized controlled trial. Obesity. 2013;21(5):904-909.

15. Harrison CL, Lombard CB, Teede HJ. Limiting postpartum weight retention through early antenatal intervention: the HeLP-her randomised controlled trial. Int J Behav Nutr Phys Act. 2014;11:134. doi: 10.1186/s12966-014-0134-8.

16. Song C, Li J, Leng J, Ma RC, Yang X. Lifestyle intervention can reduce the risk of gestational diabetes: a meta-analysis of randomized controlled trials. Obes Rev. 2016;17(10):960-969. 
17. Hoffman L, Nolan C, Wilson JD, Oats JJ, Simmons D. Gestational diabetes mellitus-management guidelines. The Australasian Diabetes in Pregnancy Society. Med J Aust. 1998;169(2):93-97.

18. Abell SK, Shorakae S, Haim D, Moreno-Asso A, Harrison C, Stepto N, De Courten $\mathrm{B}$, Teede HJ. The role of adipocytokines and inflammatory biomarkers in early identification of gestational diabetes. Diabetes Metab Res Rev. 2017. DOI: 10.1002/dmrr.2926.

19. Gillespie P, Cullinan J, O'Neill C, Dunne F, Collaborators AD. Modeling the independent effects of gestational diabetes mellitus on maternity care and costs. Diabetes Care. 2013;36(5):1111-1116.

20. Georgiou HM, Lappas M, Georgiou GM, Marita A, Bryant VJ, Hiscock R, et al. Screening for biomarkers predictive of gestational diabetes mellitus. Acta Diabetol. 2008;45(3):157-165.

21. Smirnakis KV, Plati A, Wolf M, Thadhani R, Ecker JL. Predicting gestational diabetes: choosing the optimal early serum marker. Am J Obstet Gynecol. 2007;196(4):410 e1-6; discussion e6-7.

22. Lain KY, Daftary AR, Ness RB, Roberts JM. First trimester adipocytokine concentrations and risk of developing gestational diabetes later in pregnancy. Clin Endocrinol (Oxf). 2008;69(3):407-411.

23. Lacroix M, Battista MC, Doyon M, Menard J, Ardilouze JL, Perron P, et al. Lower adiponectin levels at first trimester of pregnancy are associated with increased insulin resistance and higher risk of developing gestational diabetes mellitus. Diabetes Care. 2013;36(6):1577-1583.

24. Williams MA, Qiu C, Muy-Rivera M, Vadachkoria S, Song T, Luthy DA. Plasma adiponectin concentrations in early pregnancy and subsequent risk of gestational diabetes mellitus. J Clin Endocrinol Metab. 2004;89(5):2306-2311.

25. Nanda S, Savvidou M, Syngelaki A, Akolekar R, Nicolaides KH. Prediction of gestational diabetes mellitus by maternal factors and biomarkers at 11 to 13 weeks. Prenat Diagn. 2011;31(2):135-141.

26. Rasanen JP, Snyder CK, Rao PV, Mihalache R, Heinonen S, Gravett MG, et al. Glycosylated fibronectin as a first-trimester biomarker for prediction of gestational diabetes. Obstet Gynecol. 2013;122(3):586-594.

27. Galic S, Oakhill JS, Steinberg GR. Adipose tissue as an endocrine organ. Mol Cell Endocrinol. 2010;316(2):129-139. 


\section{Retnakaran R, Connelly PW, Maguire G, Sermer M, Zinman B, Hanley AJ.}

Decreased high-molecular-weight adiponectin in gestational diabetes: implications for the pathophysiology of Type 2 diabetes. Diabet Med. 2007;24(3):245-252.

29. Morisset AS, Dube MC, Cote JA, Robitaille J, Weisnagel SJ, Tchernof A. Circulating interleukin-6 concentrations during and after gestational diabetes mellitus. Acta Obstet Gynecol Scand. 2011;90(5):524-530.

30. Hassiakos D, Eleftheriades M, Papastefanou I, Lambrinoudaki I, Kappou D, Lavranos $\mathrm{D}$, et al. Increased maternal serum interleukin-6 concentrations at 11 to 14 weeks of gestation in low risk pregnancies complicated with gestational diabetes mellitus: development of a prediction model. Horm Metab Res. 2016;48(1):35-41.

31. Correa PJ, Vargas JF, Sen S, Illanes SE. Prediction of gestational diabetes early in pregnancy: targeting the long-term complications. Gynecol Obstet Invest. 2014;77(3):145149.

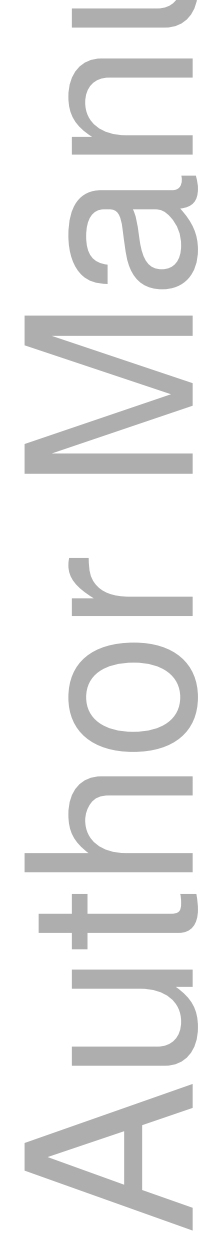


Table 1: Clinical risk prediction model for GDM

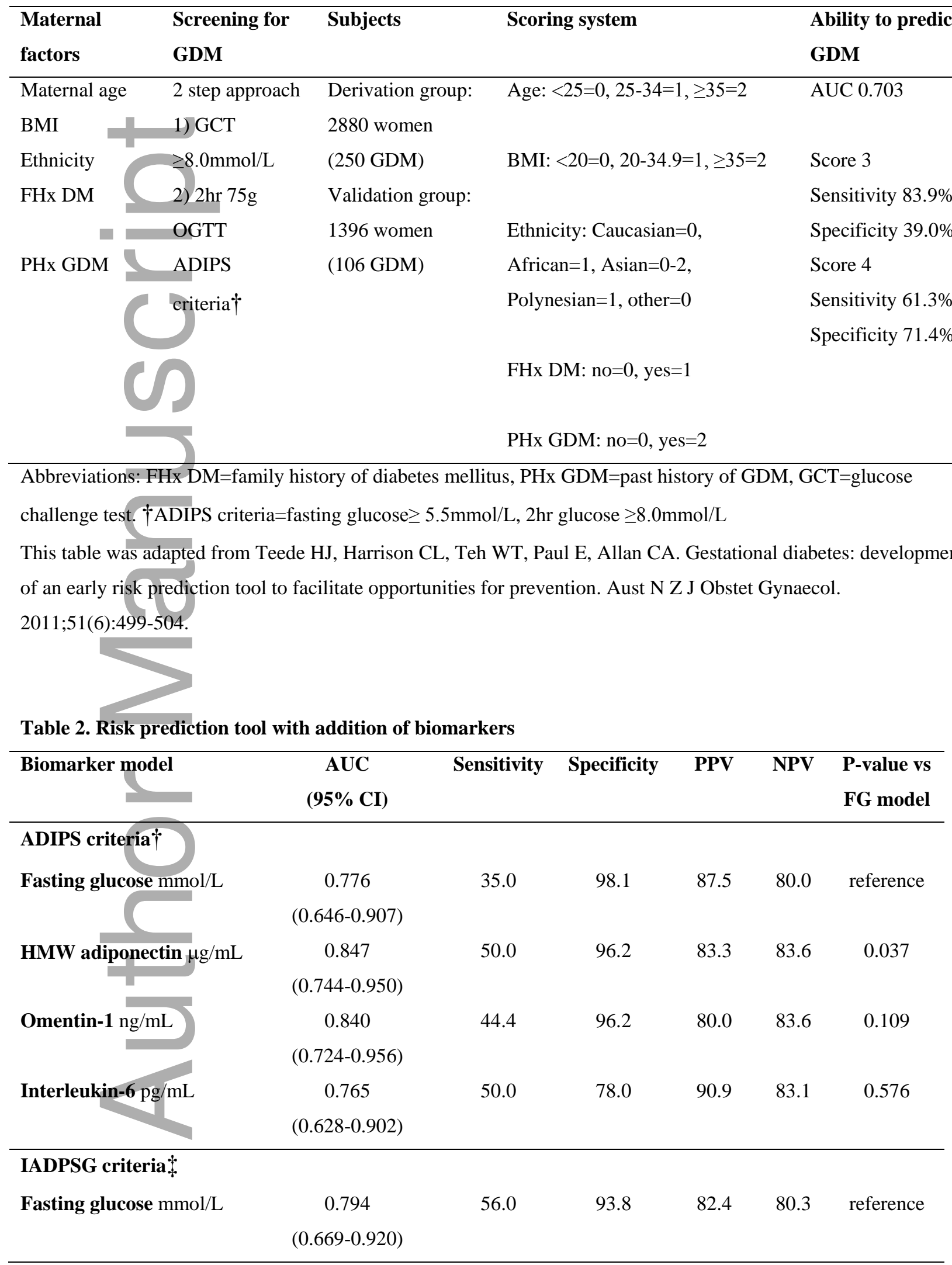




\begin{tabular}{lcccccc}
\hline HMW adiponectin $\mu \mathrm{g} / \mathrm{mL}$ & 0.838 & 64.0 & 97.9 & 94.1 & 83.9 & 0.217 \\
& $(0.735-0.942)$ & & & & & \\
Omentin-1 ng/mL & 0.781 & 52.2 & 95.8 & 85.7 & 80.7 & 0.912 \\
& $(0.654-0.907)$ & & & & & \\
Interleukin-6 pg/mL & 0.795 & 60.0 & 95.6 & 88.2 & 81.1 & 0.905 \\
& $(0.663-0.927)$ & & & & & \\
\hline
\end{tabular}

All models include maternal factors: age, BMI, ethnicity, past history of GDM and family history of diabetes mellitus (first degree relative). Each single biomarker was added to the FG tool.

Abbreviations: AUC: area under curve, $\mathrm{PPV}=$ positive predictive value, $\mathrm{NPV}=$ negative predictive value, $\mathrm{FG}=$ fasting glucose.

$\uparrow$ ADIPS criteria: fasting $\geq 5.5 \mathrm{mmol} / \mathrm{L}$ and $/$ or $2 \mathrm{hr} \geq 8.0 \mathrm{mmol} / \mathrm{L}$.

†IADPSG criteria: fasting $\geq 5.1 \mathrm{mmol} / \mathrm{L}$ and/or $1 \mathrm{hr} \geq 10.0 \mathrm{mmol} / \mathrm{L}$ and $/$ or $2 \mathrm{hr} \geq 8.5 \mathrm{mmol} / \mathrm{L}$.

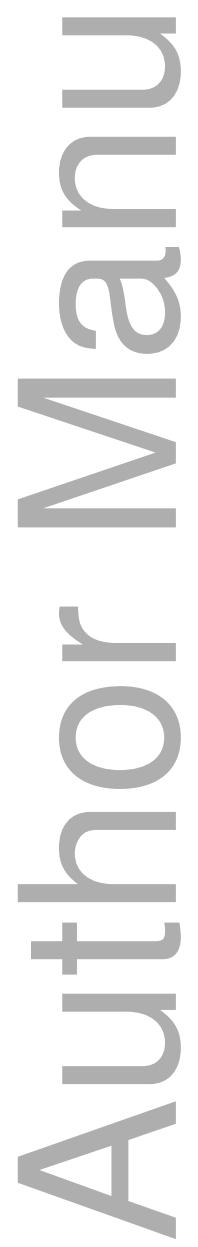


Figure 1a. ROC curve for HMW adiponectin and fasting glucose models for ADIPS criteria

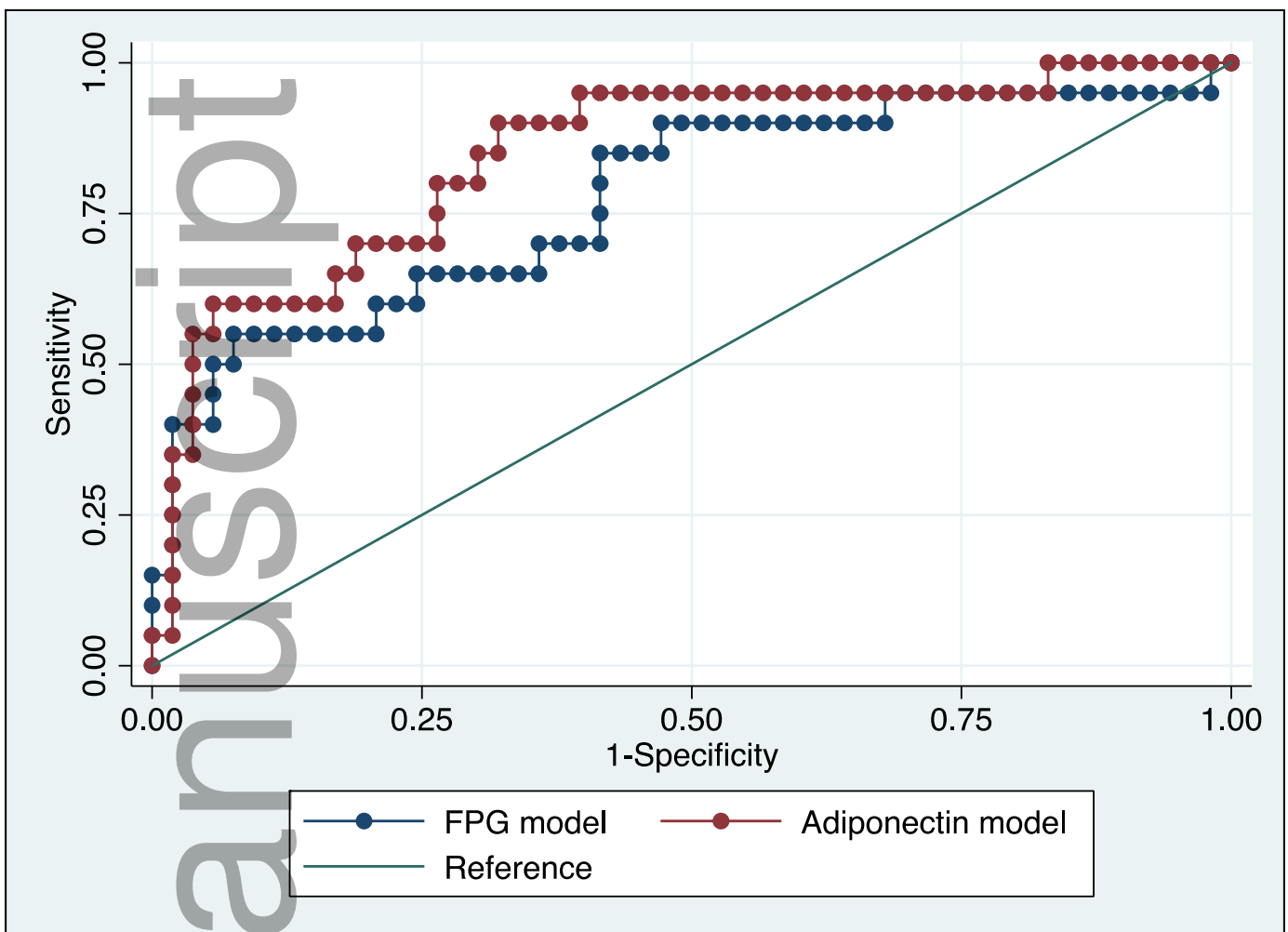

A graph of the RUC curves shows 1mproved AUC for the HMW adiponectin model (AUC O.85) compared to the fasting plasma glucose model (AUC 0.78) for prediction of ADIPS GDM.

Figure 1b. ROC curve for HMW adiponectin and fasting glucose models for IADPSG criteria

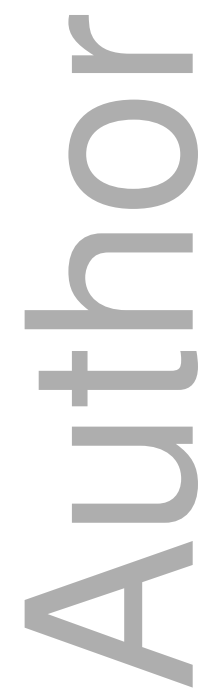

This article is protected by copyright. All rights reserved 


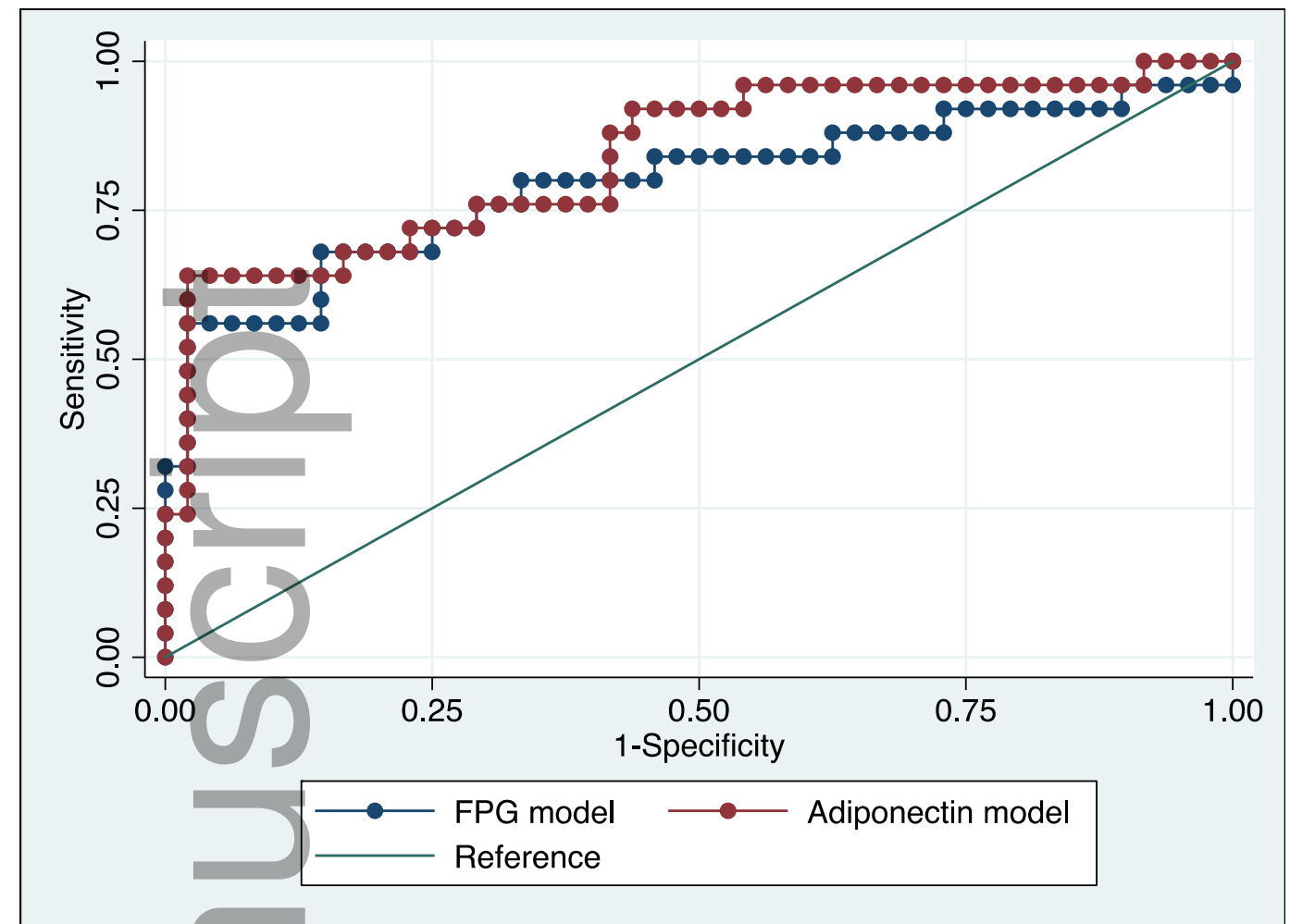

A graph of the ROC curves shows improved AUC for the HMW adiponectin model (AUC 0.84) compared to the fasting plasma glucose model (AUC 0.79) for prediction of IADPSG GDM.

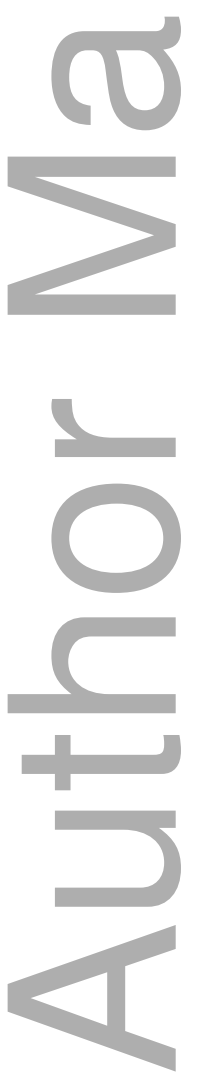

This article is protected by copyright. All rights reserved 


\section{University Library}

\section{- M M N E R VA A gateway to Melbourne's research publications}

Minerva Access is the Institutional Repository of The University of Melbourne

Author/s:

Abell, SK;Shorakae, S;Boyle, JA;De Courten, B;Stepto, NK;Teede, HJ;Harrison, CL

Title:

Role of serum biomarkers to optimise a validated clinical risk prediction tool for gestational diabetes.

Date:

2019-04

\section{Citation:}

Abell, S. K., Shorakae, S., Boyle, J. A., De Courten, B., Stepto, N. K., Teede, H. J. \& Harrison, C. L. (2019). Role of serum biomarkers to optimise a validated clinical risk prediction tool for gestational diabetes.. Aust N Z J Obstet Gynaecol, 59 (2), pp.251-257. https://doi.org/10.1111/ajo.12833.

Persistent Link:

http://hdl.handle.net/11343/285017 